\title{
A Trilogy of Submassive Pulmonary Embolism, Non-Small Cell Lung Cancer with Brain Metastasis, Kartagener's Syndrome and its Management with Aspiration Thrombectomy
}

\author{
Vinit Singh ${ }^{1}$, Dhairya Gor ${ }^{2}$, Rana Prathap Padappayil ${ }^{1}$, Ali Jaffery ${ }^{1}$, Patrick Lee ${ }^{1}$ \\ ${ }^{1}$ Internal Medicine Department, Monmouth Medical Center, Long Branch, NJ, USA \\ ${ }^{2}$ Internal Medicine Department, Jersey Shore University Medical Center, Neptune City, NJ, USA
}

Received: 10/01/2022

Accepted: $14 / 01 / 2022$

Published: 02/03/2022

How to cite this article: Singh V, Gor D, Padappayil RP, Jaffery A, Lee P. A trilogy of submassive pulmonary embolism, non-small cell lung cancer with brain metastasis, Kartagener's syndrome and its management with aspiration thrombectomy. EJCRIM 2022;9:doi:10.12890/2022_003149.

Conflicts of Interests: The Authors declare that there are no competing interests.

This article is licensed under a Commons Attribution Non-Commercial 4.0 License

\section{ABSTRACT}

Venous thromboembolism is a common cause of morbidity and mortality in cancer patients. Given the bleeding risks, determining treatment for malignancy-related thrombosis is challenging, especially in the case of submassive pulmonary embolism (PE) because the risk-benefit ratio in terms of bleeding risk is uncertain. Here we discuss the case of a 53-year-old man with Kartagener syndrome with situs inversus totalis and stage IV non-small cell lung cancer with a recent brain mass resection, presenting with dyspnoea and palpitations. The patient was diagnosed with a submassive PE and only underwent suction thrombectomy through the left internal jugular vein with excellent response. In conclusion, when treating submassive PE, aspiration thrombectomy without thrombolysis is preferable to thrombolysis, which may be contraindicated due to the higher risk of bleeding.

\section{KEYWORDS}

Submassive pulmonary embolism, non-small cell lung cancer, bleeding risk, resection, aspiration thrombectomy

\section{LEARNING POINTS}

- Anticoagulation and thrombolysis should be started after carefully weighing the risks and benefits in cancer patients.

- In some patients with submassive pulmonary embolism (PE), thrombolysis may be contraindicated due to the increased risk of bleeding, but aspiration thrombectomy can be a preferred treatment modality.

- Further research evaluating the risks and benefits of different therapeutic approaches for submassive PE could aid in determining the best course of action and establishing treatment guidelines.

\section{INTRODUCTION}

Venous thromboembolism (VTE) is one of the most common causes of morbidity and mortality in cancer patients, with a 12-month cumulative incidence after cancer diagnosis of $2.3 \%$, which is 8.5 times that of the general population ${ }^{[1]}$. According to a recent study, over the last two decades the annual incidence of VTE in cancer patients has risen threefold, with a sixfold increase in individuals receiving chemotherapy or targeted therapy ${ }^{[1]}$.

Pulmonary embolism (PE) can be categorized as massive, submassive or low-risk PE, with long-term mortality rates of $71.4 \%, 44.5 \%$ and 
28.1\%, respectively ${ }^{[2]}$. Submassive PE or intermediate-risk PE is PE in a haemodynamically stable patient with significant right ventricular dysfunction ${ }^{[3]}$. The best way to manage this group is unclear ${ }^{[4]}$. Catheter-based therapies have recently been employed to treat highrisk and intermediate-risk $\mathrm{PE}^{[4]}$. However, based on the guidelines, catheter-aided thrombus removal is only recommended in patients with acute PE and hypotension associated with a high risk of bleeding, failed thrombolysis therapy, or shock with insufficient time for thrombolytic therapy ${ }^{[5]}$. Moreover, as different malignancies are associated with different bleeding risks and comorbidities, employing a generic approach is challenging.

We present a case of submassive PE in the setting of Kartagener syndrome, with underlying stage IV non-small cell lung cancer (NSCLC) with recent brain metastases resection, treated only by aspiration thrombectomy without any systemic anticoagulation or thrombolysis.

\section{CASE DESCRIPTION}

A 53-year-old man with Kartagener syndrome with situs inversus totalis and stage IV NSCLC with metastatic brain tumour resection 20 days before presentation attended the emergency department complaining of acute worsening of underlying shortness of breath for 8 hours. The patient was managed on osimertinib for his stage IV EGFR-positive NSCLC. The patient denied any chest pain but had occasional palpitations with vital signs at presentation showing a heart rate of $129 \mathrm{bpm}$, blood pressure $108 / 69 \mathrm{mmHg}$, and spO ${ }_{2}$ of $89 \%$ on 6 litres of oxygen.

The relevant blood work results are given in Table 1. Chest x-ray showed known dextrocardia with a large area of consolidation involving the mid to lower left lung, which was improved compared with previous chest CT scans consistent with his left-sided NSCLC. EKG showed sinus tachycardia with right axis deviation with the S1Q3T3 pattern compared with the EKG 1 month previously. ACT pulmonary angiogram was performed, showing significant bilateral PE in the main pulmonary arteries (Fig. 1). Bilateral deep venous thrombosis involving the right femoral, peroneal and left common femoral veins was revealed after venous Doppler ultrasonography. An echocardiogram showed flattening of the interventricular septum (also seen on CT pulmonary angiography, Fig. 2), an elevated pulmonary artery pressure (PAP) of $56 \mathrm{mmHg}$, and significant tricuspid regurgitation with a velocity of $345.4 \mathrm{~cm} / \mathrm{sec}$, indicating right heart strain. Considering patient imaging findings, and elevated troponin levels, the patient was categorized as having submassive PE. He was not considered a candidate for thrombolysis due to a recent craniotomy with anterior frontal lobe mass resection for brain metastases.

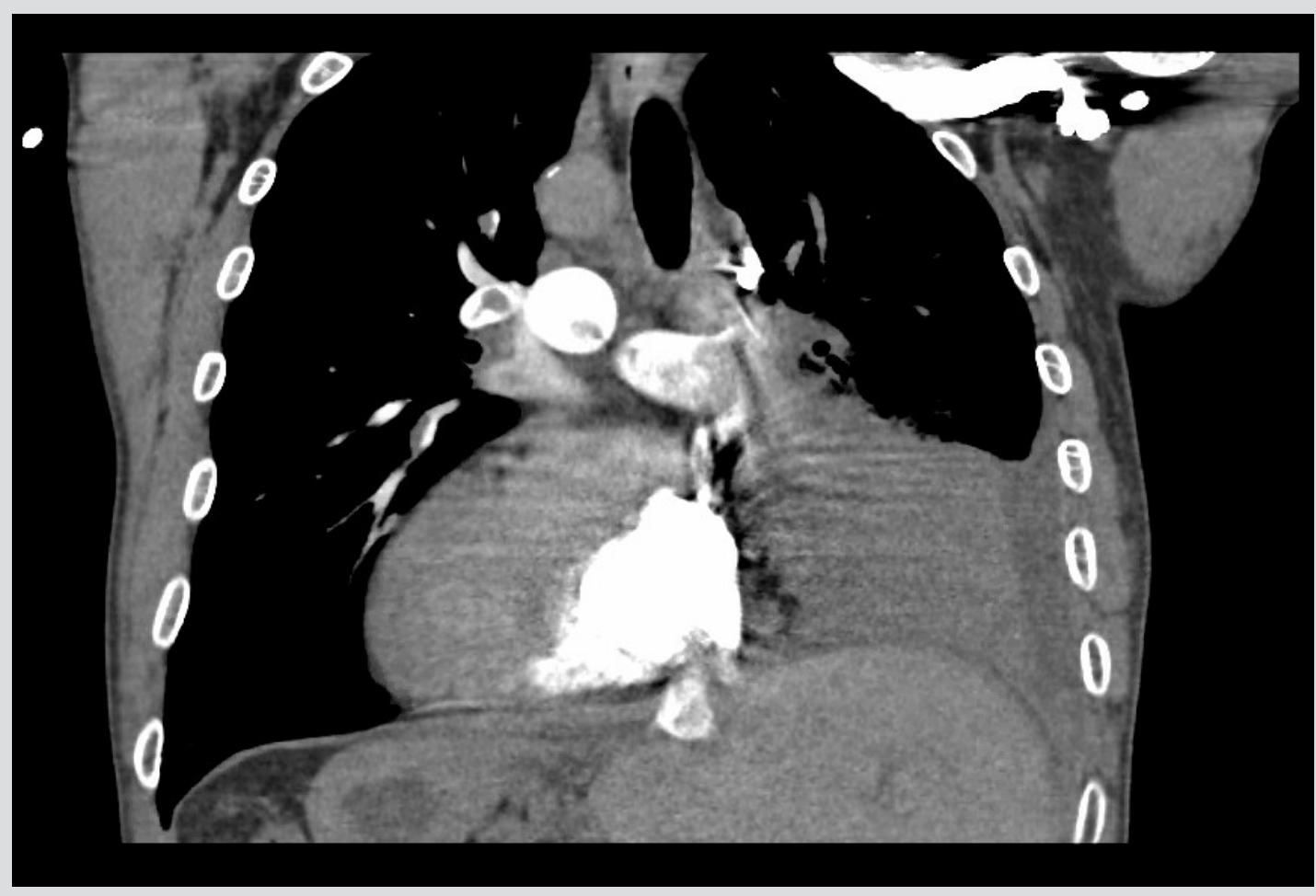

Figure 1. CT pulmonary angiogram showing the right side of the heart with remnants of emboli in the right pulmonary artery, left pulmonary artery and main pulmonary artery in the setting of dextrocardia with Kartagener syndrome 

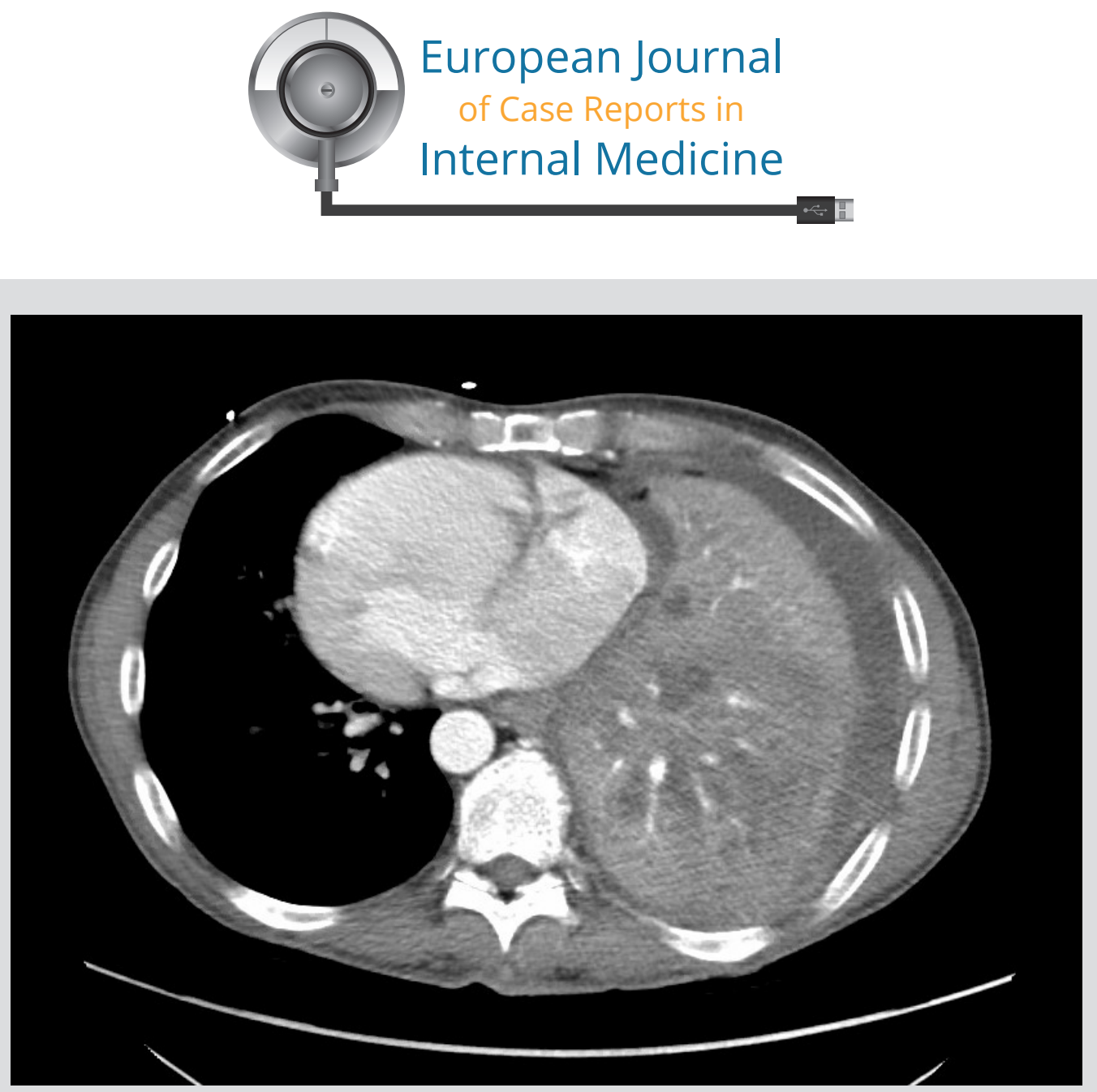

Figure 2. CT pulmonary angiogram showing contrast in the left ventricle with flattening of the interventricular septum representing strain on the left side of the heart in the setting of dextrocardia with Kartagener syndrome

\begin{tabular}{|c|c|}
\hline Parameter & Value \\
\hline WBC & $26.1 \mathrm{~K} / \mu \mathrm{l}$ \\
\hline Hb & $13.7 \mathrm{~g} / \mathrm{dl}$ \\
\hline Platelets & $127,000 / \mu \mathrm{l}$ \\
\hline Neutrophils & $84 \%$ \\
\hline Band & $9 \%$ \\
\hline Procalcitonin & $0.07 \mathrm{ng} / \mathrm{ml}$ \\
\hline Lactic acid & $3.2>2.1>2 \mathrm{mmol} / \mathrm{l}$ \\
\hline BUN & $26 \mathrm{mg} / \mathrm{dl}$ \\
\hline Creatinine & $0.73 \mathrm{mg} / \mathrm{dl}$ \\
\hline
\end{tabular}

\begin{tabular}{|c|c|}
\hline Parameter & Value \\
\hline PT & 10.4 seconds \\
\hline INR & 1 \\
\hline PTT & 26.2 seconds \\
\hline AST & $43 \mathrm{U} / \mathrm{I}$ \\
\hline ALT & $43 \mathrm{U} / \mathrm{l}$ \\
\hline ALP & $56 \mathrm{U} / \mathrm{l}$ \\
\hline Troponin-I & $0.921>1.371>0.998 \mathrm{ng} / \mathrm{ml}$ \\
\hline BNP & $28 \mathrm{pg} / \mathrm{ml}$ \\
\hline
\end{tabular}

Three consecutive troponin-I values were recorded $6 \mathrm{~h}$ apart until they started trending downwards, and three lactic acid values were recorded $4 \mathrm{~h}$ apart until they reached a value of 2 , as shown.

Table 1. Biochemical parameters at the time of presentation 
The patient was started on a heparin drip for anticoagulation after Neurosurgery consultation. Considering the patient risk of bleeding after recent surgery, suction thrombectomy using a penumbra device was employed. The patient had Kartagener syndrome, which had resulted in dextrocardia anatomy due to situs inversus. The procedure was done via the left internal jugular vein and large saddle emboli were removed en bloc. The pulmonary arteriograms before and after the procedure are compared in Fig. 3.

A follow-up echocardiogram after thrombectomy showed the PAP pressure had dropped from 56 to $43 \mathrm{mmHg}$, and the TR velocity had dropped from 345.4 to $302.7 \mathrm{~cm} / \mathrm{sec}$. On day 2 after the procedure, the patient's oxygen requirements were reduced to a 3-litre nasal cannula and he was discharged on rivaroxaban on postoperative day 5 . He continued his treatment with osimertinib with two follow-up MRIs, which showed a stable resection cavity in the left frontal lobe. Three months later, lower limb Doppler ultrasound revealed that the clots had resolved, leading to the removal of an IVC filter 6 months after it was first implanted. The patient continued to be treated with osimertinib for stage IV NSCLC and rivaroxaban for VTE risk.
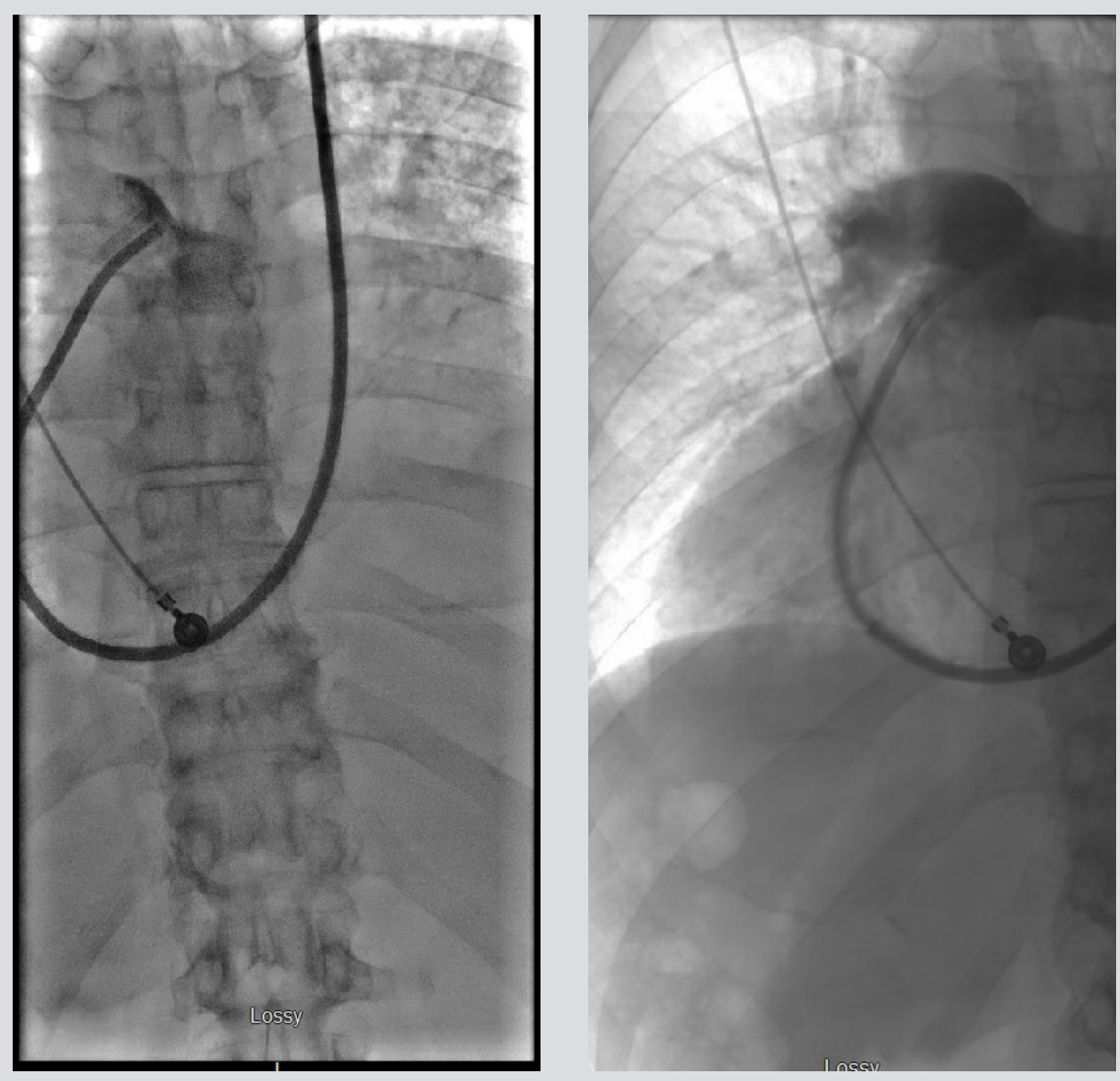

Figure 3. Pulmonary arteriogram showing (left image) decreased contrast diffusion in the pulmonary arterial circulation before embolectomy, and (right image) established pulmonary arterial circulation after embolectomy from the right lower lobe, right upper lobe pulmonary artery with penumbra thrombectomy device

\section{DISCUSSION}

Anticoagulation remains the mainstay of treatment, with fibrinolysis, catheter-assisted embolectomy, surgical embolectomy, and IVC filter implantation among the more advanced options ${ }^{[6]}$. Because there are few randomized controlled trials (RCTs) to guide the treatment of submassive PE, the decision to pursue advanced therapy or remain with anticoagulation alone must be determined individually ${ }^{[6]}$. To establish the mortality benefits and related bleeding hazards, researchers looked at 16 RCTs comparing thrombolytic treatment and anticoagulants ${ }^{[7]}$. Thrombolytics were related to decreased mortality (OR $\left.0.48,95 \% \mathrm{Cl} 0.25-0.92\right)$ but higher significant bleeding risks (OR 3.19, 95\% Cl 2.07-4.0) in patients with intermediate $\mathrm{PE}^{[7]}$. Further investigation has shown that thrombolytic therapy for submassive $\mathrm{PE}$ reduces mortality, but data for individuals at risk of bleeding are limited ${ }^{[7,8]}$. Hence our patient would have qualified for thrombolysis based on the presentation. However, thrombolysis or anticoagulation posed a higher risk of bleeding due to the patient's recent brain mass surgery. While there is no consensus on the best treatment, a few trials have shown encouraging results with thrombectomy. Consequently, aspiration thrombectomy was chosen as the treatment of choice for our patient.

Aspiration thrombectomy in PE has been shown to decrease the right-sided heart strain, quantified as right-to-left ventricular diameter 
ratio (RV/LV) and PAP. For instance in the FLARE trial, a multicentre prospective study, the FlowTriever catheter device was used for catheter embolectomy in submassive PE patients and showed a $25 \%$ drop in the 48-hour RV/LV ratio, with $p<0.0001^{[4]}$. In addition, in an expert analysis by McDaniel[9], the FLARE trial's RV/LV ratio change was compared with those seen in other trials, which showed that the shift in RV/LV ratio was equivalent to other thrombolysis trials and superior to anticoagulation alone ${ }^{[4,9]}$. Furthermore, the incidence of adverse events in the FLARE trial was comparable to those found with anticoagulation ${ }^{[4,9]}$. Nonetheless, no direct comparison studies have been conducted.

Another prospective multicentre study ${ }^{[10]}$ investigated the efficacy and safety of the Indigo aspiration device in patients with submassive $\mathrm{PE}$. The investigators found a mean reduction of $27.3 \%(95 \% \mathrm{Cl} 24.83-29.67 \%, p<0.0001)$ in the RV/LV ratio, an average reduction in systolic PAP of $4.3 \mathrm{mmHg}(95 \% \mathrm{Cl} 2.6-5.9 \mathrm{mmHg}, 7.9 \%$ reduction, $p<0.0001)$, and a low major adverse event rate of $1.7 \%(95 \% \mathrm{Cl}$ $0.0-4.0 \%, p<0.0001$ ) following use of the device. Consistent with the outcome of this trial, PAP was reduced by $23 \%$ in our patient after aspiration thrombectomy.

The efficiency of combining mechanical thrombectomy with thrombolysis has also been investigated; however, there is no evidence of a significant increase in effectiveness with the addition of thrombolysis ${ }^{[11]}$. A retrospective study of 25 patients with high-risk PE treated with percutaneous mechanical thrombectomy (PMT) over 10 years from 2005 showed no significant variations in heart rate, blood pressure or peak systolic tricuspid pressure gradient in patients who received PMT alone versus PMT with thrombolysis ${ }^{[11]}$. In addition, patients who received thrombolysis received more blood transfusions than those who did not ( $50 \%$ vs. $12 \%, p=0.04$ ), although there was no statistically significant difference in bleeding. In our case, thrombectomy alone resulted in an excellent outcome with no bleeding complications. As a result, enhanced case reporting, such as ours, can help elucidate the effectiveness and risks of aspiration thrombectomy in patients with submassive PE who are at risk of bleeding.

\section{REFERENCES}

1. Mulder FI, Horváth-Puhó E, van Es N, van Laarhoven HWM, Pedersen L, Moik F, et al. Venous thromboembolism in cancer patients: a population-based cohort study. Blood 2021 Apr 8;137(14):1959-1969. Available from: https://pubmed.ncbi.nlm.nih.gov/33171494/

2. Gupta R, Ammari Z, Dasa O, Ruzieh M, Burlen JJ, Shunnar KM, et al. Long-term mortality after massive, submassive, and low-risk pulmonary embolism. Vasc Med 2020 Apr 1;25(2):141-149. Available from: https://pubmed.ncbi.nlm.nih.gov/31845835/

3. Konstantinides SV, Torbicki A, Agnelli G, Danchin N, Fitzmaurice D, Galiè N, et al. 2014 ESC guidelines on the diagnosis and management of acute pulmonary embolism. Eur Heart J 2014 Nov 14;35(43):3033-3069, 3069a-3069k. Available from: https://pubmed.ncbi.nlm.nih.gov/25173341/

4. Tu T, Toma C, Tapson VF, Adams C, Jaber WA, Silver M, et al; FLARE Investigators. A prospective, single-arm, multicenter trial of catheter-directed mechanical thrombectomy for intermediate-risk acute pulmonary embolism: the FLARE Study. JACC Cardiovasc Interv 2019 May 13;12(9):859-869. Available from: https://pubmed.ncbi.nlm.nih. gov/31072507/

5. Kearon C, Akl EA, Ornelas J, Blaivas A, Jimenez D, Bounameaux H, et al. Antithrombotic therapy for VTE disease: CHEST guideline and expert panel report. Chest 2016 Feb 1;149(2):315-352. Available from: https://pubmed.ncbi.nlm.nih.gov/26867832/

6. Piazza G, Goldhaber SZ. Management of submassive pulmonary embolism. Circulation 2010;122(11):1124-1129. Available from: https://www.ahajournals.org/doi/ abs/10.1161/circulationaha.110.961136

7. Chatterjee S, Chakraborty A, Weinberg I, Kadakia M, Wilensky RL, Sardar P, et al. Thrombolysis for pulmonary embolism and risk of all-cause mortality, major bleeding, and intracranial hemorrhage: a meta-analysis. JAMA 2014 Jun 18;311(23):2414-2421. Available from: https://pubmed.ncbi.nlm.nih.gov/24938564/

8. Meyer G, Vicaut E, Danays T, Agnelli G, Becattini C, Beyer-Westendorf J, et al. Fibrinolysis for patients with intermediate-risk pulmonary embolism. N Engl J Med 2014 Apr 10;370(15):1402-1411.

9. McDaniel MC. FLARE: mechanical thrombectomy for intermediate-risk PE. Am Coll Cardiol Jul 312018 [accessed 12 Sep 2021]. Available from: https://www.acc.org/latestin-cardiology/articles/2018/07/31/08/38/flare-mechanical-thrombectomy-for-intermediate-risk-pe

10. Sista AK, Horowitz JM, Tapson VF, Rosenberg M, Elder MD, Schiro BJ, et al. Indigo aspiration system for treatment of pulmonary embolism: results of the EXTRACT-PE trial. JACC Cardiovasc Interv Feb 8 2021;14(3):319-329. Available from: https://pubmed.ncbi.nlm.nih.gov/33454291/

11. Bunc M, Steblovnik K, Zorman S, Popovic APP. Percutaneous mechanical thrombectomy in patients with high-risk pulmonary embolism and contraindications for thrombolytic therapy. Radiol Oncol 2020 Feb 14;54(1):62. Available from: https://www.ncbi.nlm.nih.gov/pmc/articles/PMC7087421/ 